\title{
Caracterización de enfermedad metastásica con PET/ CT en cáncer de mama en etapificación y con recidiva postratamiento
}

\author{
Dres. David Ladrón de Guevara H, Patricia Guzmán F.
}

Departamento de Radiología, Clínica Las Condes, Santiago, Chile.

Characterization of metastatic disease in recently diagnosed breast cancer and relapsed breast cancer (after treatment), with PET / CT.

\begin{abstract}
Purpose. To describe metastatic disease detected by PET/CT in breast cancer (BC), and to evaluate the relative contribution of PET and CT analyzed separately. Patients and Method. We defined two groups of patients with $B C:$ 1) recently diagnosed with no treatment, 2) with relapse after treatment. We described findings which are visible exclusively with $C T$ and exclusively with PET. Results. In recently diagnosed patients $(n=17) 88 \%$ show lymphadenopathies, $29 \%$ bone metastases (BM), $17 \%$ lung metastases, $17 \%$ hepatic metastases, and $11 \%$ other localizations. For relapsed patients $(n=35)$ these percentages were $54 \%, 62 \%, 34 \%, 31 \%$ and $28 \%$, respectively. CT detected more lung nodules and sclerotic bone lesions than PET. PET detected more lymphadenopathies, medullary bone and hepatic lesions than CT. There were synchronous cancers in $6 \%$ of recently diagnosed patients and in $11 \%$ of relapsed patients. Conclusion. BC patients show mainly lymph nodal and bone metastasis. The PET/CT hybrid study detected more lesions than PET and CT analyzed separately.
\end{abstract}

Key words: Cancer, Breast, Metastases, PET, Relapse.

Resumen: Objetivo. Describir la enfermedad metastásica detectada por PET/CT en cáncer de mama (CM) y estimar el aporte relativo del PET y del CT interpretados por separado. Pacientes y método. Se separaron dos grupos de pacientes con CM: 1) en etapificación 2) ya tratados con recidiva al PET/CT. Se describieron las lesiones encontradas exclusivamente con PET y exclusivamente con CT. Resultados. Los pacientes en etapificación ( $n=17$ ) presentaron $88 \%$ adenopatías, $29 \%$ metástasis óseas $(M O), 17 \%$ pulmonares, $17 \%$ hepáticas y $11 \%$ en otras localizaciones. Para los pacientes en seguimiento $(n=35)$ estos porcentajes fueron de $54 \%, 62 \%, 34 \%, 31 \%$ y 28\%, respectivamente. El CT detectó más nódulos pulmonares y MO escleróticas que el PET. EI PET detectó más adenopatías, MO medulares y lesiones hepáticas. Se encontró cáncer sincrónico en $6 \%$ de los pacientes en etapificación y $11 \%$ en seguimiento. Conclusión. Las pacientes con CM presentaron principalmente metástasis ganglionares y óseas. El estudio híbrido PET/CT detectó más lesiones que el PET y el CT analizados por separado.

Palabras clave: Cáncer, Mama, Metástasis, PET, Recidiva.

Ladrón de Guevara, et al. Caracterización de enfermedad metastásica con PET/CT en cáncer de mama en etapificación y con recidiva postratamiento. Rev Chil Radiol 2013; 19(1): 21-28.

Correspondencia: Dr. David Ladrón de Guevara Hernández. / dlg@clc.cl

Trabajo recibido el 04 de marzo de 2013, aceptado para publicación el 20 de marzo de 2013

\section{Introducción}

EI PET/CT (Positron Emission Tomography/Computed Tomography) ha demostrado gran utilidad en la etapificación y seguimiento de pacientes con cáncer de mama y ha ido incorporándose paulatinamente en los distintos protocolos y algoritmos de estudio de esta patología ${ }^{(1)}$. Su capacidad de detectar metástasis ganglionares y a distancia con mayor sensibilidad que cualquier otro método de imagen, y de explorar todo el cuerpo de una sola vez, son sus ventajas más reconocidas. Estas cualidades se traducen en una mayor cantidad de lesiones detectadas y un campo de estudio corporal más extenso, con detección más precoz y en sitios no sospechados con las técnicas 
de imagen convencionales.

El objetivo de este trabajo es describir el compromiso ganglionar y metastático a distancia detectado con PET/CT F18-FDG en pacientes con cáncer de mama, tanto en pacientes en etapificación, como en aquellos ya tratados y que sufren una recidiva, y estimar el aporte relativo del PET y del CT interpretados por separado en la detección de lesiones.

\section{Pacientes y método}

Pacientes: Se seleccionaron los PET/CT con F18-FDG realizados a pacientes con diagnóstico de cáncer de mama realizados entre enero de 2009 y diciembre de 2011, separándose en dos grupos clínicos: 1) pacientes en etapificación con diagnóstico reciente, y que no han recibido tratamiento; y 2) pacientes con cáncer de mama ya tratado, en que el PET/CT mostró recidiva (re-etapificación). Los pacientes sin compromiso ganglionar ni metástasis fueron excluidos.

Realización del PET/CT: Todos los PET/CT fueron efectuados en un equipo GE Discovery STE de 16 canales, con adquisición de CT para corrección de atenuación y localización, seguido por adquisición 3D de imágenes metabólicas para el mismo campo. Los PET/CT corporales comprendieron un campo desde base de cráneo hasta raíz de muslos, y se realizaron 60 minutos después de la inyección de F18-FDG calculada según $\mathrm{Kg}$ de peso. La gran mayoría de las pacientes se efectuó además una tomografía computada (TC) de tórax, abdomen y pelvis en el mismo equipo, inmediatamente posterior a la adquisición del PET/CT y previa administración de contraste iodado endovenoso. Se llevó a cabo una entrevista con cada paciente para conocer sus antecedentes y dar las indicaciones de preparación para el examen. Dicha entrevista fue realizada por un médico o un tecnólogo médico, según la disponibilidad. Antes de administrar la dosis ev de F18-FDG se midió glicemia capilar mediante hemoglucotest en todos los pacientes, con valor de corte de $200 \mathrm{mg} / \mathrm{dl}$ para poder realizar el examen. En caso de niveles mayores se instaló suero fisiológico para hidratación y se controló glicemia hasta alcanzar un nivel adecuado. La infusión de insulina rápida no fue necesaria en ningún paciente.

Análisis semicuantitativo: Se cuantificó la captación de los ganglios comprometidos y metástasis a distancia en todos los casos, utilizando el índice SUVmax (Standarized uptake value maximum), el que se obtiene dibujando un área de interés sobre la lesión, con cálculo automático de la actividad del vóxel más captante, resultando en una medida de actividad por volumen, corregida por la dosis inyectada y peso del paciente ${ }^{(2)}$. Se consignó el índice SUVmax de cada lesión metastásica, registrando aquella que mostró la mayor captación para cada localización (ganglionar, ósea, hepática etc).

Interpretación de las imágenes: Las imágenes del PET y la TC fueron evaluadas de manera separada por dos observadores expertos, consignando la presencia de lesiones metastásicas ganglionares y a distancia visibles por el método correspondiente. Las lesiones de cada órgano o sistema fueron contabilizadas y caracterizadas una a una. Un número igual o mayor a 40 lesiones por órgano o sistema fue considerado como "múltiples" y contabilizada como 40 lesiones en dicho órgano. Luego ambos observadores analizaron el estudio híbrido PET/CT y la TC con contraste, comparando lesión a lesión y consignando consensuadamente los falsos negativos de cada técnica. Se definió "falso negativo" (FN) como una lesión visualizada por un método (por ejemplo TC), pero no por otro (en este caso PET), correspondiendo a un FN de la segunda técnica. Para el análisis comparativo PET vs TC de lesiones abdominales (hepáticas, esplénicas, etc) y ganglionares se incluyó sólo los casos en que se realizó TC con contraste endovenoso. Considerando el estudio híbrido completo (PET/CT + TC con contraste yodado endovenoso) y la interpretación conjunta de ambos observadores, se describió la enfermedad metastásica ganglionar y a distancia del grupo en etapificación y del grupo en re-etapificación. El "Gold Standard" para determinar falsos negativos y positivos fue la biopsia o el seguimiento de las lesiones. Se definió "falso positivo" como una lesión sospechosa al PET o a la TC que resultó benigna en la biopsia o resección. La detección de una segunda malignidad o segundo tumor primario no fue considerado falso positivo.

Confirmación de las lesiones metastásicas: Todos los tumores primarios mamarios fueron biopsiados y/o resecados, con estudio histopatológico disponible en todos los casos. La correlación histológica ganglionar fue posible sólo en los casos con vaciamiento axilar y/o resección dirigida. En caso de extenso compromiso axilar y/o metástasis a distancia múltiples, la confirmación de la naturaleza maligna de las lesiones se efectuó indirectamente al observar respuesta al tratamiento o bien progresión en PET/CT, TC o resonancia magnética de control. En caso de lesión única sospechosa de metástasis al PET/CT, ésta fue siempre biopsiada y/o resecada, o seguida con controles de imágenes para determinar su naturaleza.

Análisis de los datos: Se describió la distribución y número de lesiones ganglionares y a distancia detectadas por el PET/CT (estudio híbrido completo), tanto en el grupo en etapificación como en el grupo en re-etapificación. Se realizó un estudio comparativo simple de ambas técnicas (PET y TC) para detección de compromiso ganglionar y de metástasis a distancia, determinando los falsos negativos por paciente y por número de lesiones para cada una, según se mencionó previamente. 
Estudio estadístico: Aunque el presente estudio es esencialmente descriptivo, se utilizaron algunas pruebas estadísticas como el t-test para comparar los índices SUVmax entre las distintas lesiones, y Chi cuadrado para evaluar tablas de contingencia.

\section{Resultados}

Descripción de enfermedad metastásica

Los pacientes en etapificación ( $n=17$, promedio edad 54 años, rango 29-69 años) correspondieron en un $70 \%$ a carcinoma ductal y $30 \%$ a carcinoma lobulillar. Presentaron al PET/CT un 88\% de compromiso ganglionar, 29\% metástasis óseas (MO), 17\% pulmonares (MP), 17\% hepáticas $(\mathrm{MH})$ y $11 \%$ en otras localizaciones (OL). Se encontró una alta concordancia ( $p<0,0001$, coeficiente de contingencia: 0,51) en axila entre PET/CT y resultado histopatológico en aquellos que se realizaron vaciamiento axilar, con 35 ganglios verdaderos positivos del PET, 37 verdaderos negativos del PET, 20 falsos negativos del PET (16 de ellos micrometastasis) y 1 falso positivo del PET (Tabla I).

Los 36 pacientes en seguimiento con sospecha de recidiva (re-etapificación) correspondieron a tumores de histología ductal en $66 \%$ y lobulillar en $34 \%$. Un paciente finalmente no se contabilizó en este grupo luego que se comprobó que las adenopatías hipercaptantes inguinales y obturadoras visibles en el PET/CT resultaron de origen inflamatorio crónico

Tabla I. PET FDG vs histopatología en ganglios axilares resecados en pacientes con cáncer de mama sometidas a vaciamiento axilar.

\begin{tabular}{|l|lrl|}
\multicolumn{2}{|c|}{ PET } \\
\hline \multirow{4}{*}{ Histopatología } & + & + & - \\
\cline { 2 - 4 } & - & 35 & 20 \\
& - & 1 & 37 \\
\hline P $<0,0001$, Coeficiente de contingencia: 0,51) \\
\hline
\end{tabular}

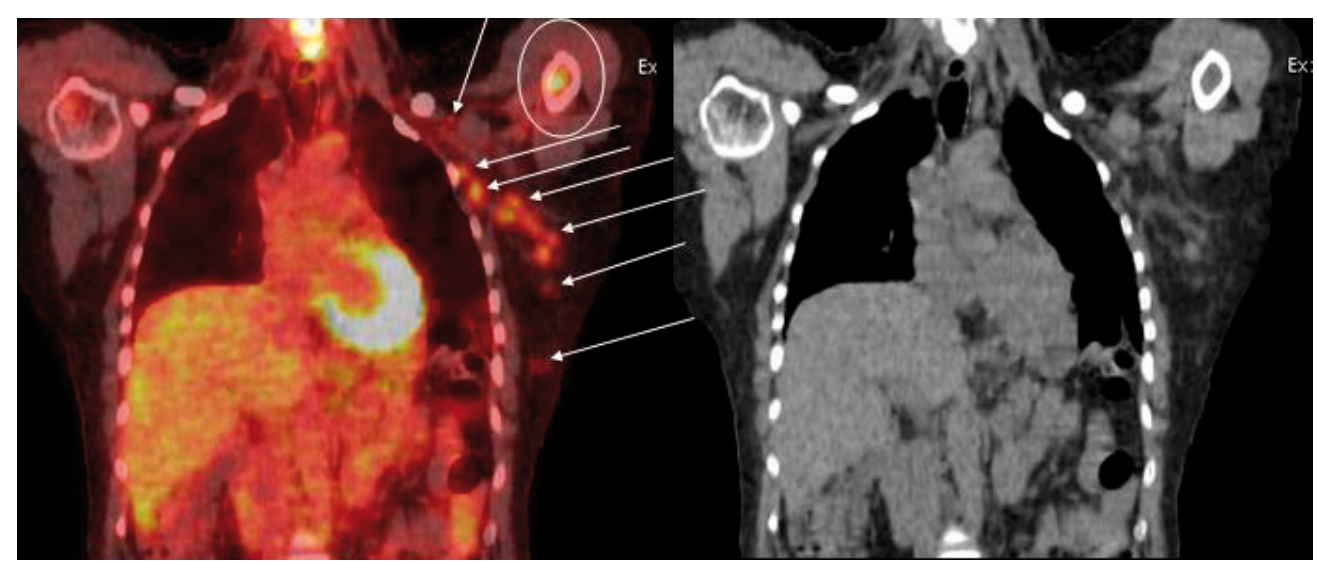

en la histología, correspondiendo a un falso positivo del PET. Los PET/CT de este grupo $(n=35$, promedio edad 54 años, rango 34-82 años) mostraron $54 \%$ de recidiva ganglionar, $62 \% \mathrm{MO}, 34 \% \mathrm{MP}, 31 \%$ $\mathrm{MH}, 8 \%$ suprarrenales y $28 \% \mathrm{OL}$. Una paciente con nódulo hipercaptante en la mama operada que fue sospechoso de recidiva local al PET, fue confirmada histológicamente como necrosis grasa.

La distribución de frecuencia de metástasis en ambos grupos clínicos se muestra en la Tabla II.

\section{PET vs TC analizados por separado}

Pacientes en etapificación: De los 75 ganglios hipermetabólicos detectados por el PET, sólo 47 fueron positivos en la TC, con $29 \mathrm{FN}$ de la técnica (Sensibilidad de la TC: 61,8\%). La mayor parte de los FN de la TC correspondieron a pequeños ganglios axilares ipisilaterales (n:26) (Figura 1). Un falso positivo del TC se observó en axila ipsilateral.

De las 76 metástasis óseas presentes en cinco pacientes el PET detectó 71 lesiones y la TC 69, con 5 FN del PET en un paciente (4 lesiones escleróticas y una lítica), y 7 FN de la TC en dos pacientes, ambos con lesiones medulares (Figura 2).

De las 81 metástasis hepáticas presentes en tres pacientes, el PET las detectó todas, y la TC 80, con un FN de esta última técnica.

Respecto a las 44 metástasis pulmonares el PET presentó $8 \mathrm{FN}$ en tres pacientes, sin FN para la TC. Las lesiones no visualizadas con PET correspondieron a nódulos pequeños, menores de $6 \mathrm{~mm}$.

Pacientes en reetapificación: De los 148 ganglios sospechosos al PET, 90 fueron anormales en la TC (Sensibilidad de la TC: 60,8\%). Los falsos positivos de la TC ocurrieron principalmente en hilios pulmonares ( $n: 17)$, región cervical baja (n:12) y mediastino (n:10). Un falso positivo de la TC ocurrió en una adenopatía cervical, que no se resecó pero evolucionó como benigna.

De las 535 metástasis óseas en 22 pacientes, el PET detectó 529 (6 FN en un paciente con lesiones escleróticas), y la TC 454 (81 FN en ocho pacientes correspondientes a 70 lesiones medulares y 11 líticas incipientes).

Figura 1. Numerosas pequeñas adenopatías axilares y retropectorales izquierdas hipermetabólicas al PET (flechas), algunas de las cuales son negativas a la TC. Metástasis medular ávida de FDG en humero izquierdo (círculo) sin traducción en TC. Captación normal de FDG en corazón e hígado. 


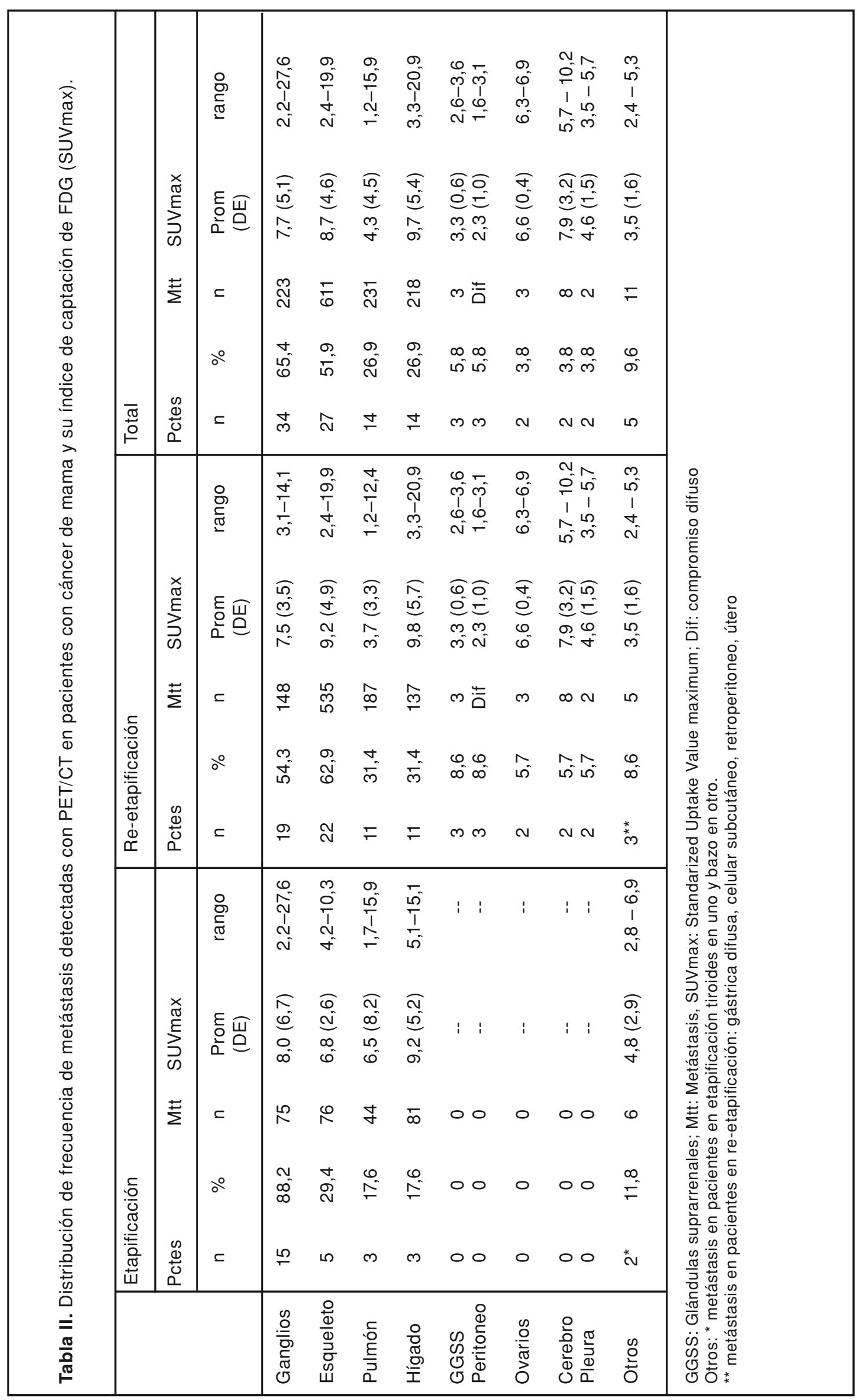


De las 137 metástasis hepáticas, el PET no vio 3 en un paciente, y la TC no vio 2 en dos pacientes (Figura 3 ).

Respecto a las 195 metástasis pulmonares encontradas, el PET no vio 14 en cuatro pacientes, y la TC no vio 6 en dos pacientes. Este último grupo de falsos negativos de la TC correspondió a lesiones nodulares hipercaptantes ubicadas dentro de una atelectasia o condensación, en bandas densas descritas como residuales o bien nódulos de base pleural o cisural pasados por linfonodos.

Las metástasis en otras localizaciones fueron de franca menor frecuencia, predominando glándulas suprarenales en $8 \%$ y peritoneo en $8 \%$ del grupo en reetapificación. EI PET detectó lesiones secundarias en útero y en tiroides no sospechadas con TC (ambas confirmadas histológicamenete) (Figura 4).
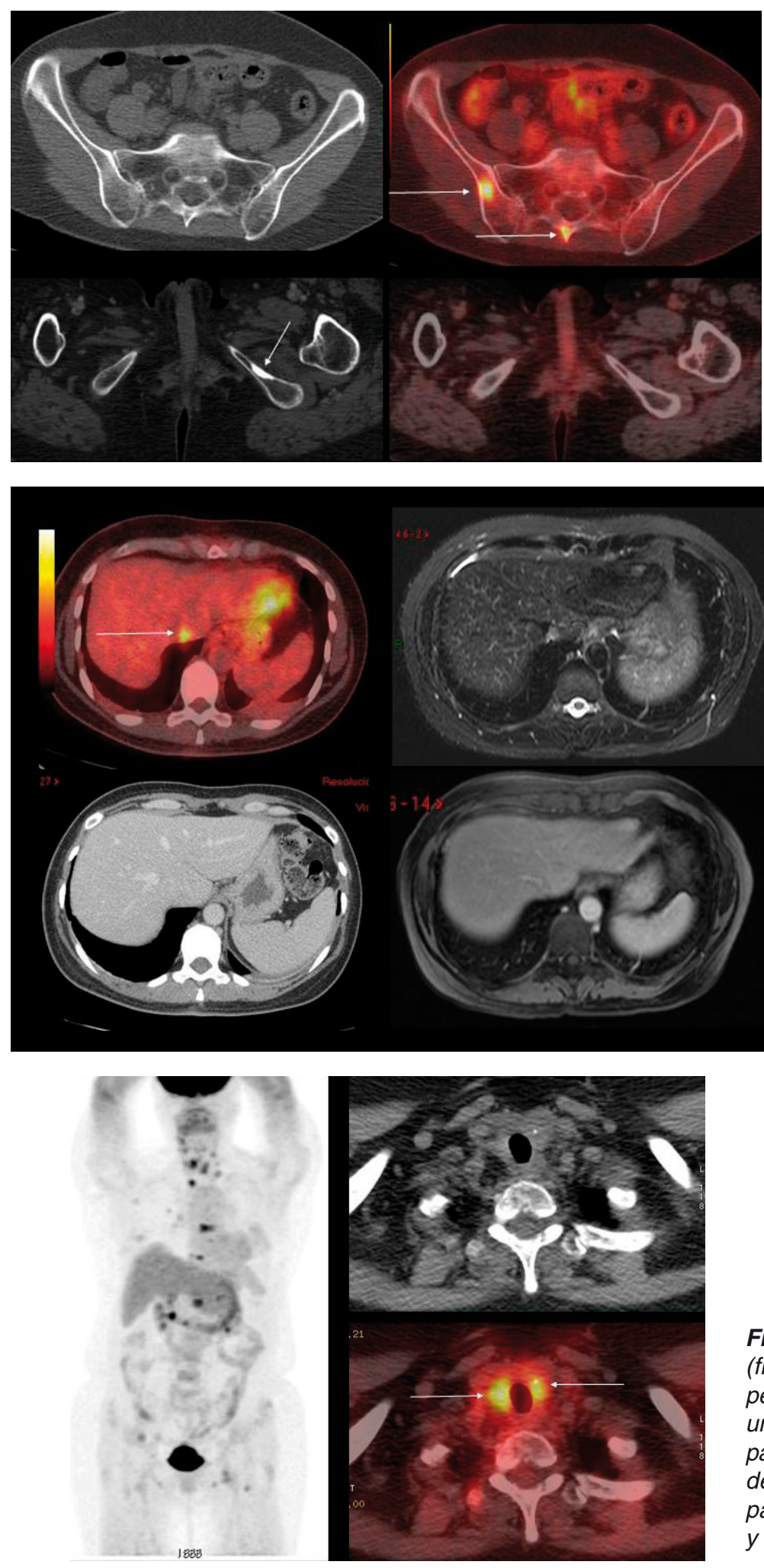

Figura 2. Dos lesiones óseas medulares hipercaptantes al PET (flechas) sin representación a la TC (fotos superiores). Las fotos inferiores muestran una lesión esclerótica al TC sin hipercaptación evidente al PET.

Figura 3. Lesión hepática hipermetabólica al PET, no visible a la TC y de difícil visualización en la $R M$.

Figura 4. Metástasis tiroideas hipercaptantes (flechas largas). La existencia de adenopatías peritirodeas (flechas cortas) hicieron sospechar un segundo primario tiroideo, pero la anatomía patológica demostró múltiples implantes tiroideos de cáncer ductal indiferenciado de mama. La paciente presentaba también metástasis óseas y en pulmón derecho. 
Caracterización de las lesiones metastásicas

En pacientes en etapificación el compromiso ganglionar fue principalmente axilar ipsilateral $(66 \%$ de los casos), seguido por cervical bajo (14\%) y mamario interno (10\%). Una minoría tuvo diseminación a ganglios supraclaviculares (5\%) o axilares contralaterales (5\%). El promedio de ganglios por paciente fue de cinco (rango 0-13 ganglios). EI SUVmax promedio en ganglios comprometidos fue de 8,0 (DE: 6,7, rango: 2,8-27,6).

El grupo en re-etapificación presentó múltiples localizaciones ganglionares, principalmente en mediastino (22\%), hilios pulmonares (15\%), supraclaviculares (13\%) y axilares ipsilaterales (13\%). El promedio de lesiones por paciente fue de 4,3 (rango 0 -30 ganglios). EI SUVmax promedio fue de 7,5 (DE: 3,5 , rango: 3,1-15,6).

Las metástasis óseas fueron principalmente escleróticas (380) y medulares (110), con menor proporción de lesiones líticas (69) y mixtas (52). La localización fue predominantemente en esqueleto axial, con especial compromiso de esternón y columna vertebral. De los pacientes con metástasis óseas, el $20 \%$ en etapificación (1/5) y el 40\% en reetapificación (9/22) presentaban lesiones múltiples (40 ó más lesiones). La captación no fue significativamente diferente entre el grupo en etapificación (SUVmax promedio: 6,8, DE: 2,6) y en re-etapificación (SUVmax promedio: 9,2, DE: 4,8).

Las lesiones hepáticas fueron múltiples en cuatro pacientes, dos de ellos en etapificación, y únicas en cuatro individuos, tres de ellos en re-etapificación. No hubo diferencias significativas en la captación entre el grupo en etapificación (SUVmax promedio:9,2, DE: 5,2 ) y re-etapificación (SUVmax promedio:9,8, $\mathrm{DE}: 5,6)$.

Las metástasis pulmonares fueron múltiples en sólo dos pacientes, ambos en reetapificación. La gran mayoría correspondieron a nódulos sólidos, exhibiendo hipercaptación al PET con tamaños desde
$6 \mathrm{~mm}$. La captación promedio de estas lesiones en el grupo completo de pacientes fue SUVmax: 9,6 (DE: 5,4, rango: 1,1-20,9).

Las restantes localizaciones fueron menos frecuentes tanto en los pacientes en etapificación (tiroides y bazo) como en re-etapificación (glándulas suprarrenales, peritoneo, ovario, etc), como se muestra en la Tabla II.

\section{Detección de un segundo tumor primario}

Se observó cáncer sincrónico al de mama en $6 \%$ (1/17) de los pacientes en etapificación (Ca de células renales). EI PET/CT detectó una segunda neoplasia en $11 \%$ (4/35) de los pacientes en seguimiento (Cáncer páncreas, tiroides, peritoneal primario y trompa uterina). Uno de estos casos se muestra en la Figura 5.

\section{Discusión}

Protocolos consensuados actuales mencionan el PET F18-FDG en la etapificación inicial de cáncer de mama desde la etapa IIIA como una alternativa al estudio tradicional ${ }^{(1)}$. Su aporte principal radica en su alto rendimiento en la detección de enfermedad ganglionar $(\mathrm{N})$ y de metástasis a distancia $(\mathrm{M})$. Por otro lado, su utilidad en el seguimiento y reetapificación en pacientes ya tratados ha sido largamente demostrada en múltiples publicaciones ${ }^{(3,4)}$. Su elevado rendimiento en la detección de lesiones secundarias ha cambiado el paradigma en la evaluación de la enfermedad metastásica del cáncer de mama, redefiniendo la frecuencia y distribución de las lesiones respecto de las técnicas tradicionales ${ }^{(3)}$.

En este estudio nos enfocamos en describir la distribución de la enfermedad metastásica en pacientes que se realizaron PET/CT con F18-FDG en nuestro centro, considerando separadamente dos grupos de pacientes: los recientemente diagnosticados y aquellos ya tratados con recidiva, evaluando además la utilidad relativa de los dos componentes del estudio híbrido, el PET y la TC.

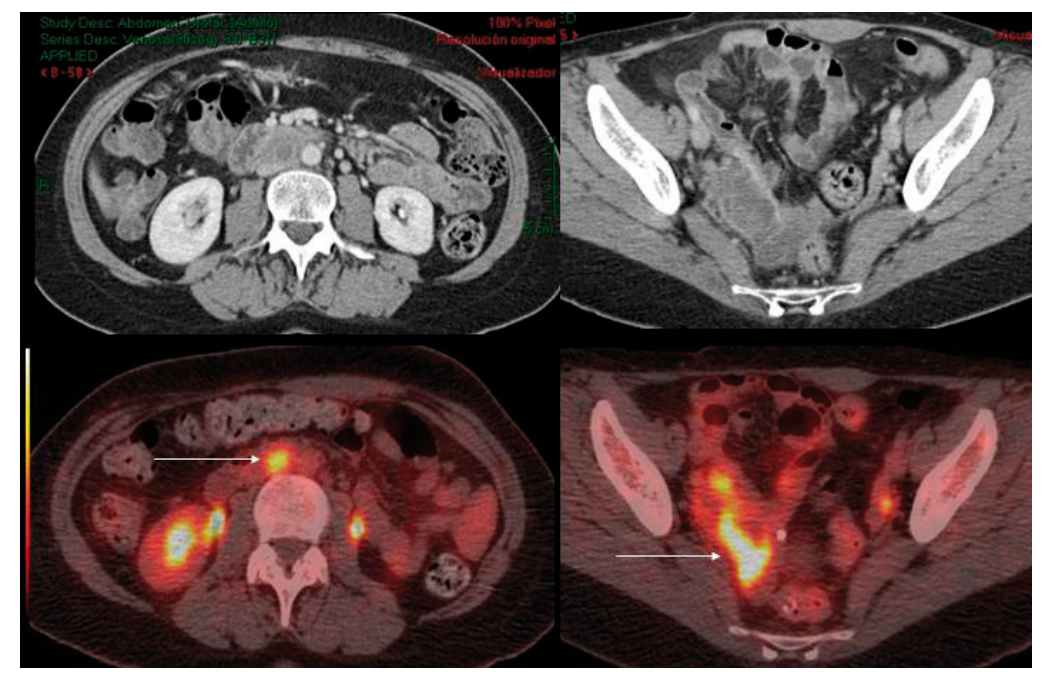

26
Figura 5. Cáncer de trompa derecha con adenopatías retroperitoneales pesquisado en una paciente en control por cáncer de mama tratado hace 9 años. 
En nuestro estudio se demostró el valor complementario del PET y la TC en la detección de lesiones, observándose un mayor aporte relativo de la TC en pulmón y del PET en la evaluación esquelética y en localizaciones atípicas como útero, tiroides y bazo. Ambas técnicas resultaron complementarias también en hígado.

El compromiso ganglionar en el grupo en etapificación involucró principalmente a la axila ipsilateral, y el PET/CT identificó la mayoría de las lesiones, aún cuando existieron 20 ganglios falsos negativos del PET, 16 de los cuales correspondían a micrometástasis nodales. La existencia de sólo un falso positivo indica un alto valor predictivo positivo del PET en axila. En el grupo de pacientes tratados recidivados, la distribución de las metástasis nodales se dispersó, existiendo numerosas localizaciones, las más frecuentes en mediastino, hilios pulmonares y supraclavicular.

Las metástasis a distancia más frecuentes encontradas fueron las óseas, correspondiendo al $29 \%$ de la muestra en etapificación. Aunque la población de nuestro estudio no es representativa de aquella con cáncer recientemente diagnosticado debido a que los pacientes sin metástasis fueron excluidos, la prevalencia descrita se acerca al $31-42 \%$ publicado en grandes series ${ }^{(5,6)}$. Nuestra menor prevalencia de enfermedad metastásica esquelética pudiera ser explicada en parte por el criterio de selección de los pacientes a los que se les solicita el PET/CT, y al diagnóstico cada vez más precoz del cáncer debido a estrictas políticas de screening. En el cohorte de pacientes en seguimiento post tratamiento, la ocurrencia de metástasis óseas fue de $62 \%$, más cercana al $58 \%$ descrito para este grupo ${ }^{(6)}$.

Las características variables del tipo de lesiones óseas secundarias encontradas en el cáncer de mama, las que pueden ser de tipo lítico, mixto, blástico o medular, hacen que no exista un solo examen que las detecte todas de una sola vez. EI PET resultó mejor para ver las lesiones medulares y líticas incipientes, las que suelen no ser visibles con la TC debido a la ausencia o insuficiente destrucción ósea necesaria para aparecer como de menor densidad que el hueso normal. Por otro lado, la TC mostró superioridad en detectar las lesiones escleróticas o blásticas, las que pueden no captar glucosa marcada debido a que se asocian a menor actividad tumoral. Las metástasis mixtas, es decir con áreas líticas y escleróticas, fueron evidentes con las dos técnicas, al igual que las líticas puras. Como ya se mencionó, se observó una importante complementariedad de los componentes PET y TC en la evaluación esquelética.

Las metástasis pulmonares resultaron las segundas más frecuentes en nuestro estudio, con un $17 \%$ en el grupo en etapificación, similar a la prevalencia publicada ${ }^{(5)}$, y un $34 \%$ en el grupo en seguimiento.
La forma de presentación más frecuente es el nódulo pulmonar, la gran mayoría de las veces de tipo sólido. La captación de F18-FDG fue detectable en nódulos desde los $6 \mathrm{~mm}$, lo que es coincidente con la resolución espacial propia de los equipos PET/CT actuales. La TC mostró clara superioridad en la visualización de nódulos pulmonares respecto al PET. Sin embargo, es interesante el hecho que el PET logró pesquisar lesiones que pasan desapercibidas con la TC, como lesiones incluidas en el espesor de atelectasias, condensaciones o bandas pulmonares, o nódulos en plano cisural o pleural que simulan linfonodos.

Las metástasis hepáticas tienen una prevalencia de aproximadamente $13 \%$ en series sobre cáncer de mama recientemente diagnosticado ${ }^{(5)}$ y de hasta $61 \%$ en necropsias ${ }^{(7)}$. La primera cifra es concordante con el $17 \%$ obtenido en nuestra muestra. Cabe destacar que estas lesiones son las que mostraron tendencia a mayor captación (índice SUVmax). La detección de tres lesiones hepáticas por PET que no eran visibles en la TC, y el hecho que estas lesiones son frecuentemente únicas, constituye un punto a favor del uso de la técnica híbrida en el estudio hepático de estos pacientes.

La alta frecuencia de compromiso secundario pleural descrito en la literatura, de hasta $27 \%{ }^{(5)}$, no se reflejó en nuestra experiencia, donde sólo dos pacientes en re-etapificación la presentaron.

Se debe considerar que nuestro estudio necesariamente subestima la frecuencia de metástasis cerebrales, por cuanto no comprende el cerebro como campo de adquisición de manera habitual (toma de imágenes desde la base craneana), a menos que se sospeche previamente lesión encefálica. Esto se suma a la baja sensibilidad del PET en cerebro debido a la elevada captación fisiológica de la corteza cerebral, que suele enmascarar las lesiones. De hecho, los dos pacientes en que se detectaron metástasis cerebrales, éstas mostraron una elevada actividad metabólica, aún mayor que la del cerebro, lo que está asociado a alta agresividad de la lesión ${ }^{(8)}$.

La presencia de dos casos falsos positivos del $\mathrm{PET} / \mathrm{CT}$ (adenopatías hipercaptantes que resultaron inflamación crónica en una paciente y un foco de necrosis grasa mamaria hipercaptante en otra) reafirman el concepto de que toda lesión única hipermetabólica debe biopsiarse antes de ser considerada metástasis. La ocurrencia de segundas neoplasias primarias que pueden ser confundidas con diseminación del cáncer de mama refrenda lo antes mencionado.

\section{Bibliografía}

1. National Comprehensive Cancer Network (Revisado el 23 de Junio de 2012) En: http://www.nccn.org/ professionals/physician_gls/f_guidelines.asp\#site.

2. Ladrón de Guevara D, Pefaur R. PET/CT en cáncer pulmonar. Rev Med Chile 2010; 138: 1441-1450.

3. Radan L, Ben-Haim S, Bar-Shalom R, Guralnik L and 
Israel O. The Role of FDG-PET/CT in Suspected Recurrence of Breast Cancer. Cancer 2006;107:2545-51.

4. Rosen EL, Eubank WB, Mankoff DA. FDG PET, PET/ CT and Breast Cancer Imaging. RadioGraphics 2007; 27:S215-S229.

5. Hess KR, Varadhachary GR, Taylor SH, Wei W, Raber MN, Lenzi R, et al. Metastatic Patterns in Adenocarcinoma. Cancer 2006; 106: 1624-1633.

6. Briasoulis E, Karavasilis V, Kostadima L, Ignatiadis M, Fountzilas G, Pavlidis N. Metastatic Breast Carcinoma Confined to Bone. Portrait of a Clinical Entity. Cancer
2004; 101: 1524-1528.

7. Abrams HL, Spiro R, Goldstein N. Metastases in carcinoma. Analysis of 1000 autopsied cases. Cancer 1950; January: 74-85.

8. Ueda S, Tsuda H, Asakawa H, Shigekawa T, Fukatsu $\mathrm{K}$, Kondo $\mathrm{N}$ et al. Clinicopathological and Prognostic Relevance of Uptake Level using 18F-fluorodeoxyglucose Positron Emission Tomography/ Computed Tomography Fusion Imaging (18F-FDG PET/CT) in Primary Breast Cancer. Jpn J Clin Oncol 2008; 38: 250-258. 